\title{
Molecular-dynamics simulations of glass formation and crystallization in binary liquid metals: $\mathrm{Cu}-\mathrm{Ag}$ and $\mathrm{Cu}-\mathrm{Ni}$
}

\author{
Yue Qi, Tahir Çağın, * Yoshitaka Kimura, and William A. Goddard III* \\ Materials and Process Simulation Center, Beckman Institute (139-74), Division of Chemistry and Chemical Engineering, \\ California Institute of Technology, Pasadena, California 91125
}

(Received 7 August 1998)

\begin{abstract}
We used molecular dynamics (MD) to obtain an atomistic description of the melting, glass formation, and crystallization processes in metal alloys. These studies use the quantum Sutton-Chen many-body potentials for $\mathrm{Cu}, \mathrm{Ni}$, and $\mathrm{Ag}$ to examine the $\mathrm{Cu}_{4} \mathrm{Ag}_{6}$ and $\mathrm{CuNi}$ alloys. Using cooling rates in the range of $2 \times 10^{12}$ to 4 $\times 10^{14} \mathrm{~K} / \mathrm{s}$, we find that $\mathrm{CuNi}$ and pure $\mathrm{Cu}$ always form a face-centered-cubic (fcc) crystal while $\mathrm{Cu}_{4} \mathrm{Ag}_{6}$ always forms a glass (with $T_{g}$ decreasing as the quench rate increases). The crystal formers have radius ratios of $1.025(\mathrm{CuNi})$ and $1.00(\mathrm{Cu})$ while the glass former $(\mathrm{CuAg})$ has a ratio of 1.13 , confirming the role of size mismatch in biasing toward glass formation. [S0163-1829(99)05205-4]
\end{abstract}

\section{INTRODUCTION}

Metal glasses were prepared ${ }^{1}$ by the Duwez group at Caltech in the 1960's by cooling a CuAg eutectic at $10^{6} \mathrm{~K} / \mathrm{s}$. The understanding of the factors predisposing an alloy to glass formation have been developed sufficiently that Johnson et l. $^{2}$ have made TiZr based metal glasses at cooling rates of only $1 \mathrm{~K} / \mathrm{s}$. Factors that play an important role are a mismatch in atomic sizes (e.g., $\mathrm{Zr}$ versus $\mathrm{Ti}$ with a radius ratio of 1.10 or $\mathrm{Ag}$ versus $\mathrm{Cu}$ with a radius ratio of both 1.13 favor formation of a glass) ${ }^{3}$ and a mismatch in bond character (thus, adding $\mathrm{Be}$ or $\mathrm{B}$ plus $\mathrm{Fe}$ or $\mathrm{Ni}$ to the TiZr favors glass formation). Such amorphous metal glasses have numerous desirable properties; for instance, they are extremely ductile and resistant to corrosion. ${ }^{4}$ Despite the progress in making TiZr-based amorphous metals (which are now in commercial use), it has not yet been possible to develop amorphous metal glasses based on $\mathrm{Fe}$, or $\mathrm{Al}$, or $\mathrm{Cu}$, each of which might lead to numerous practical applications. To develop a better understanding of the factors controlling glass formation in metal alloys, we have started a program in carrying out atomistic simulations to elucidate the factors controlling the balance between crystallization and glass formation.

Molecular-dynamics (MD) simulations have previously been used to study glass formation from liquid in LennardJones systems ${ }^{5,6}$ and many realistic systems with different force fields. Among these are studies on pure metals, such as sodium, ${ }^{7}$ potassium, ${ }^{8}$ rubidium,,${ }^{9}$ nickel, ${ }^{10}$ and iron,${ }^{11}$ and binary metal-alloys including good glass formers like $\mathrm{Ni}-\mathrm{Zr},{ }^{12,13} \mathrm{Ti}-\mathrm{Al},{ }^{14}$ and $\mathrm{Ni}_{33} \mathrm{Y}_{67}{ }^{15}$

In this paper, we use molecular dynamics in conjunction with the quantum Sutton-Chen (Q-SC) force fields (FF), ${ }^{16-18}$ to examine melting and quenching of $\mathrm{CuNi}$ and $\mathrm{CuAg}$ alloys. These two model systems were particularly chosen, since $\mathrm{Cu}$ and Ag have very different sizes, making them good candidates for forming a metal glass, while $\mathrm{Cu}$ and Ni have similar sizes thus making them good candidates for forming a crystal even at high quenching rates.

In Sec. II, we summarize various details of the calculations (the Q-SC many-body FF used to describe the metal alloys and the MD approaches used here). Section III describes the results showing that $\mathrm{CuAg}$ forms a glass at all quenching rates used while $\mathrm{CuNi}$ forms a crystal at these same quenching rates.

\section{CALCULATION METHODS}

\section{A. Force-field parameters}

The total energy of the system in Sutton-Chen (SC)-type many-body FF has the following form:

$$
U_{\mathrm{tot}}=\sum_{i} U_{i}=\sum_{i}\left[\frac{1}{2} \sum_{j \neq i} D_{i j} V\left(r_{i j}\right)-c_{i} D_{i i} \rho_{i}^{1 / 2}\right],
$$

where $r_{i j}$ is the distance between atom $i$ and $j$. Here $V\left(r_{i j}\right)$ is a pairwise repulsive potential

$$
V\left(r_{i j}\right)=\left(\frac{\alpha_{i j}}{r_{i j}}\right)^{n}
$$

between atoms $i$ and $j$ (arising primarily from Pauli repulsion between the core electrons), while the metallic bonding is captured in $\rho_{i}$ a local energy density associated with the atom $i$ and defined as

$$
\rho_{i}=\sum_{j \neq i} \phi\left(r_{i j}\right)=\sum_{j \neq i}\left(\frac{\alpha_{i j}}{r_{i j}}\right)^{m} .
$$

Here $D$ sets the overall energy scale, $c_{i}$ is a dimensionless parameter scaling the attractive term relative to the repulsive term, and $\alpha$ is an arbitrary length parameter leading to a dimensionless form for $V$ and $\rho$.

Recently, we reparametrized the empirical many-body FF of the SC type ${ }^{16,17}$ for the face-centered-cubic (fcc) metals, by fitting to experimental properties such as density, cohesive energy, moduli, and phonon frequencies while including the zero-point energy (quantum) effects. ${ }^{18}$ The parameters used in this study are listed in Table I. The quantum SuttonChen (Q-SC) potential leads to accurate values for surface energies, vacancy energies, and stacking-fault energies.

We employed the following combination rules in describing the interaction between different types of atoms: 
TABLE I. Parameters for the quantum Sutton-Chen (Q-SC) force field (Ref. 17). See Eqs. (1)-(3) for explanations.

\begin{tabular}{lccccc}
\hline \hline & $\begin{array}{c}D \\
(\mathrm{meV})\end{array}$ & $c$ & $m$ & $n$ & $\begin{array}{c}\alpha \\
(\AA)\end{array}$ \\
\hline $\mathrm{Ag}$ & 4.0072 & 94.948 & 6 & 11 & 4.0691 \\
$\mathrm{Cu}$ & 5.7921 & 84.843 & 5 & 10 & 3.6030 \\
$\mathrm{Ni}$ & 7.3767 & 84.745 & 5 & 10 & 3.5157 \\
\hline \hline
\end{tabular}

$$
\begin{gathered}
D_{i j}=\sqrt{D_{i i} D_{j j}}, \\
n_{i j}=\frac{1}{2}\left(n_{i}+n_{j}\right), \\
m_{i j}=\frac{1}{2}\left(m_{i}+m_{j}\right),
\end{gathered}
$$

and

$$
\alpha_{i j}=\frac{1}{2}\left(\alpha_{i i}+\alpha_{j j}\right)
$$

There is no combination rule for $c_{i}$, since its value depends only on the type of the atom at which the local energy density or force is evaluated.

The cutoff distance for the interactions was taken as

$$
\left(R_{\text {cut }}\right)_{i j}=2 \alpha_{i j} .
$$

This cutoff distance includes up to the sixth coordination shell in the fcc metals.

Although the parameters were fitted to the properties of the crystal at low temperature, the Q-SC FF leads to accurate results for the liquid state. Furthermore, these parameters led to good accuracy for predicting transport properties, such as the shear viscosity of liquid $\mathrm{CuAu}$ alloy as a function of concentration and temperature obtained from simulations using nonequilibrium MD method. ${ }^{19}$

\section{B. Simulation methods}

The simulations in this paper are based on constant temperature, constant thermodynamic tension $(\mathrm{TtN}) \mathrm{MD}$ method. ${ }^{20}$ This combines the Nosè canonical ensemble ${ }^{21}$ with the Parrinello-Rahman variable shape size ensemble. ${ }^{22,23} \mathrm{TtN}$ MD captures very detailed microscopic information about the system, allowing us to study the phase transformation while permitting the shape and size of the cell to change. With an accurate FF it should give the values of volume, structure, energy, and other thermal properties comparable with experimental data.

The TtN MD simulations started from a cubic box with 500 atoms subject to periodic boundary conditions. To obtain the stress free reference size and shape of the unit cell, we performed 25 ps of simulation with constant enthalpy, constant thermodynamic tension $(\mathrm{HtN})$ at zero pressure. The TtN MD simulations were carried out in a series of increasing temperatures from 300 to $1500 \mathrm{~K}$ in $100 \mathrm{~K}$ increments. The final temperature of $1500 \mathrm{~K}$ is a few hundred degrees above the melting temperature. At every temperature the MD time step was taken as $1 \mathrm{fs}$ and the simulation time for determining the properties was $25 \mathrm{ps}$.

After equilibrating the structure in the liquid phase at $1500 \mathrm{~K}$, we cooled the system using different quenching rates from $1500 \mathrm{~K}$ down to $300 \mathrm{~K}$ in $100 \mathrm{~K}$ decrements in the $\mathrm{TtN}$ ensemble. To achieve the fast, intermediate, and slow cooling rates, we kept the model system at the same temperature for times of 50,25 , and $0.25 \mathrm{ps}$. This leads to cooling rates of $2 \times 10^{12}, 4 \times 10^{12}$, and $4 \times 10^{14} \mathrm{~K} / \mathrm{s}$, respectively. To ensure convergence of the results for the fastest cooling rate $(0.25$ ps per $100 \mathrm{~K})$, using the conditions at the end of each $0.25 \mathrm{ps}$ interval (for each $100 \mathrm{~K}$ drop) we performed additional 25 ps long $\mathrm{TtN}$ simulations for thermodynamic averaging.

The Hamiltonian for the $\mathrm{TtN}$ form of MD (Ray and $\operatorname{Rahman}^{20}$ ) has the form

$$
\begin{aligned}
H\left(\rho, \Pi, \mathbf{h}, P_{h}, s, P_{s}\right)= & \sum_{i}^{N} \frac{\pi_{i \alpha} G_{\alpha \beta}^{-1} \pi_{i \beta}}{2 m_{i}}+\frac{\operatorname{Tr}\left(\Pi^{\prime} \Pi\right)}{2 W}+\frac{P_{s}^{2}}{2 Q} \\
& +U(\rho, \mathbf{h})+V_{o} \operatorname{Tr}(t \epsilon)+g k_{B} T_{0} \ln (s),
\end{aligned}
$$

where $\left(\rho_{i}, \pi_{i}\right)$ are the generalized coordinates and conjugate momenta of particle $i, U$ is the potential energy, and $\left(h_{\alpha \beta}, \Pi_{\alpha \beta}\right)$ are the coordinates and momenta of the periodic simulation cell. The prime is used to indicate matrix transposition.

The $\mathbf{h}$ matrix is a dynamical variable describing the shape and size of the simulation cell, $\mathbf{h}=(\mathbf{a}, \mathbf{b}, \mathbf{c})$, where $\mathbf{a}, \mathbf{b}$, and $\mathbf{c}$ are the three unit vectors describing the periodic unit cell. The Nosè scaling variable and its conjugate momenta are denoted as $\left(s, P_{s}\right)$ while $T_{0}$ is the reservoir temperature in the canonical ensemble. The mass parameters $Q$ and $W$ are used in defining the kinetic energy terms for Nosé variable and cell variables. The strain tensor is given by

$$
\boldsymbol{\epsilon}=\frac{1}{2}\left(\mathbf{h}_{0}^{\prime-1} \mathbf{G h}_{o}^{-1}-\mathbf{I}\right),
$$

where $\mathbf{G}$ is the metric tensor

$$
\mathbf{G}=\mathbf{h}^{\prime} \mathbf{h} .
$$

In the analysis, we have used radial distribution function (RDF) and a structure factor evaluated for various $\mathbf{K}$ vectors; for completeness, we define them below. The RDF is calculated as

$$
g(r)=\sum_{i} \sum_{j \neq i} \delta\left(r_{j}-r_{i}\right) .
$$

The structure factor $S(\mathbf{K})$ for a given $\mathbf{K}$ vector is calculated as

$$
S(\mathbf{K})=\frac{1}{N}\left|\sum_{j} e^{2 \pi i \mathbf{K} \cdot \mathbf{r}_{j}}\right|^{2},
$$

where $\mathbf{K}$ is a reciprocal-lattice vector of the simulation cell,

$$
\mathbf{K}=\mathbf{h}^{\prime-1}\left(n_{a}, n_{b}, n_{c}\right)
$$




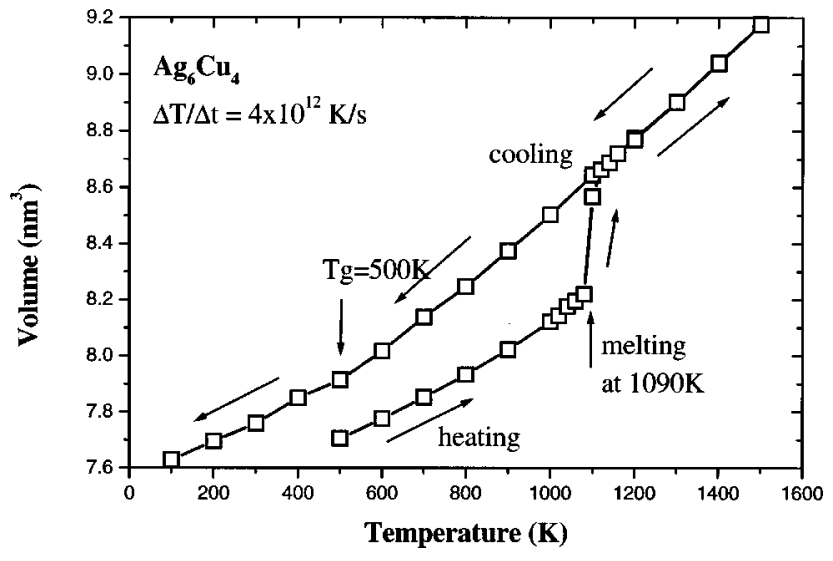

FIG. 1. Average volume of $\mathrm{Ag}_{6} \mathrm{Cu}_{4}$ during heating and cooling at a rate of $4 \times 10^{12} \mathrm{~K} / \mathrm{s}$. This shows a melting temperature of 1090 $\mathrm{K}$ (experiment $1053 \mathrm{~K}$ ). Upon cooling, the liquid is supercooled until $500 \mathrm{~K}$ where it transforms to a glass.

where $\left(n_{a}, n_{b}, n_{c}\right)$ are integers. For specific $\mathbf{K}$ vectors $S(\mathbf{K})$ leads to $N$ (the total number of atoms in the simulated system) for a perfect crystal.

\section{The choice of alloy systems}

In the $\mathrm{Ag}-\mathrm{Cu}$ alloy system, a eutectic exists at 60.1 at. \% $\mathrm{Ag}$. Indeed a metallic glass was made experimentally in 1960 by rapidly quenching the molten alloy. ${ }^{1}$ Because this propensity to form a glass is due to the difference in atomic size $\left[\left(R_{\mathrm{Ag}}-R_{\mathrm{Cu}}\right) / R_{\mathrm{Cu}}=0.13\right]$, we chose $\mathrm{Cu}_{4} \mathrm{Ag}_{6}$ as the model glass former.

On the other hand, $\mathrm{Cu}$ and $\mathrm{Ni}$ have very similar atomic size $\left[\left(R_{\mathrm{Cu}}-R_{\mathrm{Ni}}\right) / R_{\mathrm{Cu}}=0.025\right]$ so that $\mathrm{CuNi}$ is expected to form a random fcc structure at low temperature. Indeed (experimental) rapid quenching of liquid $\mathrm{CuNi}$ or $\mathrm{Cu}$ from the melt has not yet led to formation of a glass.

We carried out a series of TtN MD simulations as a function of temperature to simulate the heating and cooling processes. The cooling rate was controlled by limiting the simulation time at each temperature.

The RDF and volume were averaged over the full trajectory for each temperature. The structure factor was also calculated from the trajectory file.

\section{RESULTS AND DISCUSSION}

The models we studied were random fcc alloys of $\mathrm{Ag}(60 \%) \mathrm{Cu}(40 \%)$ and $\mathrm{Cu}(50 \%) \mathrm{Ni}(50 \%)$, and pure $\mathrm{Cu}$

\section{A. $\mathrm{CuAg}$}

Figure 1 shows the variation of the volume as $\mathrm{Ag}_{6} \mathrm{Cu}_{4}$ is heated and cooled. The large jump in volume in the temperature range of 1000 to $1200 \mathrm{~K}$ for the heating process is due to the melting of the Ag-Cu alloy. In order to obtain a more refined estimate of $T_{\text {melt }}$, we used a smaller increment in temperature, namely $20 \mathrm{~K}$, from 1000 to $1200 \mathrm{~K}$. This leads to a theoretical melting temperature, $T_{\text {melt }}=1090 \mathrm{~K}$, in reasonable agreement with experimental melting temperature of
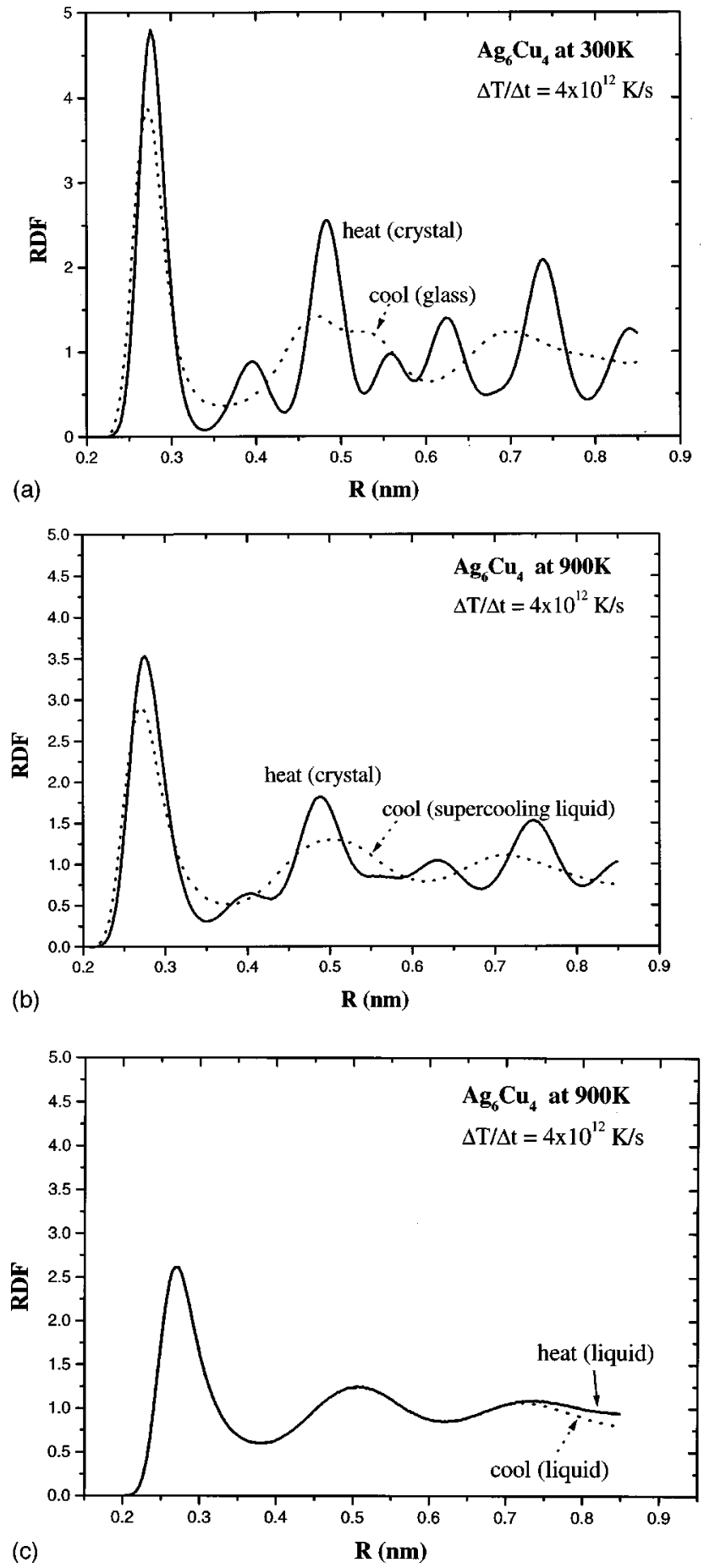

FIG. 2. Radial distribution function (RDF) of $\mathrm{Ag}_{6} \mathrm{Cu}_{4}$ during the heating and cooling processes (at the rate of $4 \times 10^{12} \mathrm{~K} / \mathrm{s}$ ). (a) 300 $\mathrm{K}$, heat denotes a random fcc crystal in the heating cycle and cool denotes the metallic glass in the end of cooling cycle. (b) $900 \mathrm{~K}$, heat (heating cycle) structure is still a random fcc and cool (cooling cycle) structure is a supercooled liquid. (c) At $1200 \mathrm{~K}$, both (structures from heating and cooling cycles) are in liquid state.

$1053 \mathrm{~K}$. One reason for the melting temperature being a bit high is that our system is homogeneous without a free surface. In addition, we started with a perfect crystal; given the rapid rate of heating, the system might not have had time to generate an equilibrium distribution of defects, thus leading to a slightly higher $T_{\text {melt }}$. 

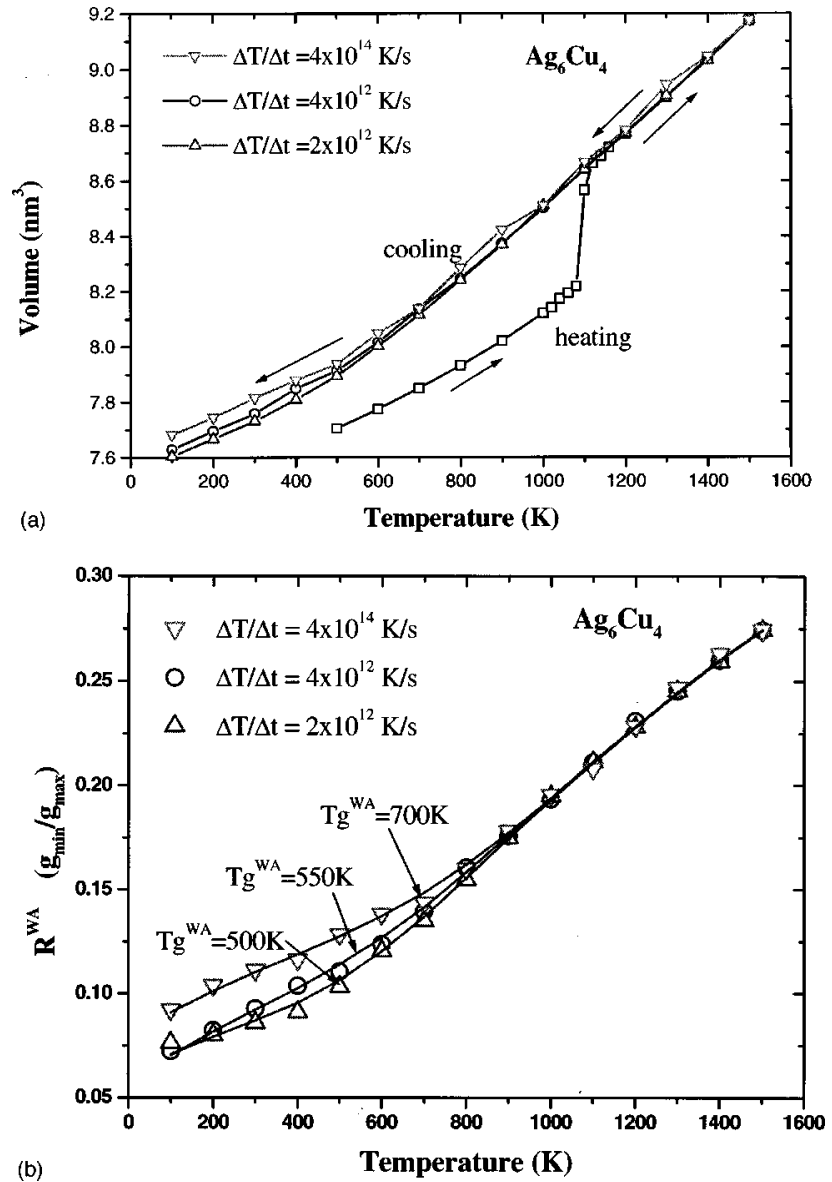

FIG. 3. Dependence of glass transition temperature on cooling rate. (a) Volume versus temperature for $\mathrm{Ag}_{6} \mathrm{Cu}_{4}$ obtained from three different quenching rates. (b) Wendt-Abraham parameter $R$ versus temperature. The calculated $T_{g}^{\mathrm{WA}}$ for three different cooling rates are 500,550 , and $700 \mathrm{~K}$.

In contrast to heating we see that cooling leads to a continuous change in volume. However, for $\mathrm{Cu}_{4} \mathrm{Ag}_{6}$, the slope of the volume versus temperature curve decreases below 500 $K$. This is a sign of glass formation. Since the glass is a frozen liquid, the change in configurational entropy vanishes. Thus, the derivative of entropy with respect to pressure is the derivative of volume with respect to temperature.

Figure 2 shows the RDF of the model structure during the heating and cooling processes. The RDF shows an fcc crystal structure as the sample is heated from 300 to $900 \mathrm{~K}$. However, at $1200 \mathrm{~K}$ (just above melting) the emergence of broad peaks shows that the structure has melted. The sample was heated to $1500 \mathrm{~K}$ and then cooled back to $1200 \mathrm{~K}$, leading to the same structure as for heating, indicating a stable liquid state. Cooling to $900 \mathrm{~K}$, from RDF we still see the structure of a liquid, in fact a supercooled liquid. However, after cooling to $300 \mathrm{~K}$, we observe that the second peak of RDF is split. This splitting of the second peak is a well-known characteristic feature in the RDF of a metallic glass. Thus, quenching the $\mathrm{Ag}_{6} \mathrm{Cu}_{4}$ alloy from the liquid to $300 \mathrm{~K}$ at the rate of $4 \times 10^{12} \mathrm{~K} / \mathrm{s}$ leads to a metallic glass.
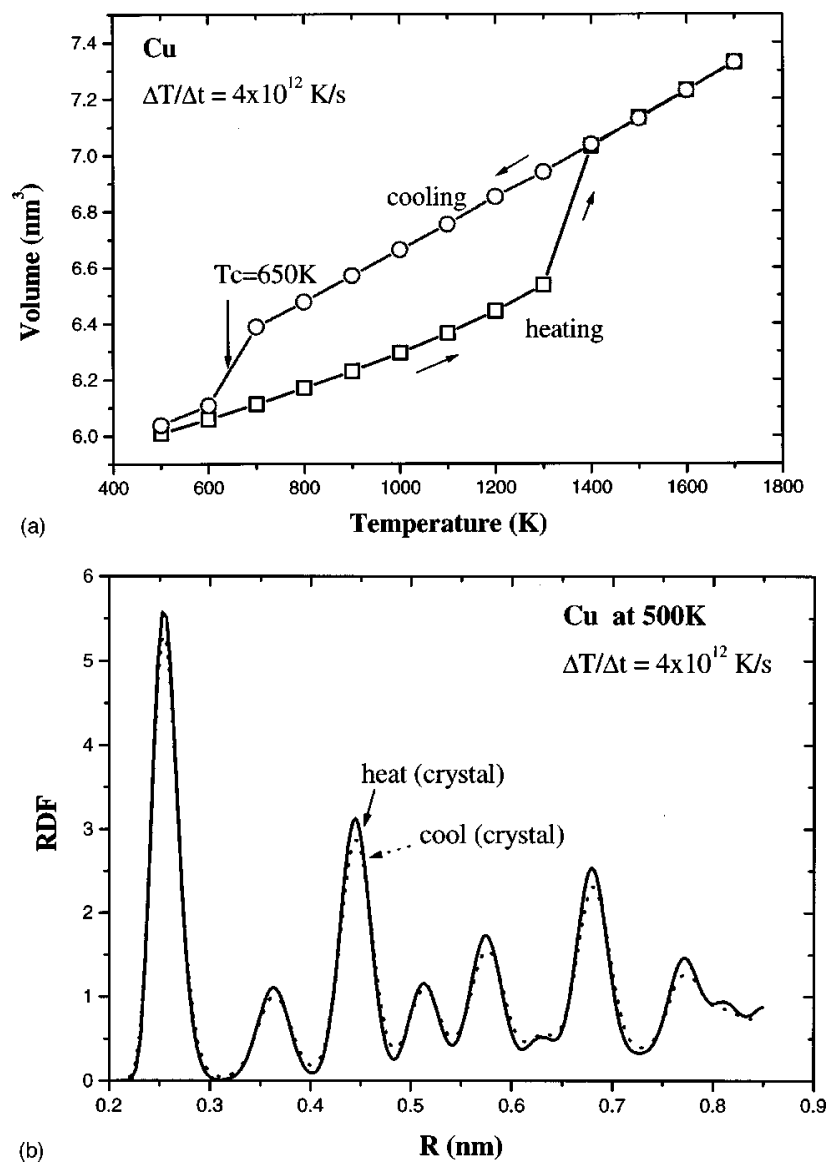

FIG. 4. Melting and cooling: quenching rate of pure $\mathrm{Cu}$ at 4 $\times 10^{12} \mathrm{~K} / \mathrm{s}$ leads to crystallization at $600 \mathrm{~K}$. (a) Volume versus temperature during heating and cooling cycles. (b) RDF at $500 \mathrm{~K}$ for structures obtained during the heating and cooling cycles.

We have considered several cooling rates to investigate its effect on the glass transition temperature $T_{g}$. Figure 3(a) shows the volume versus temperature curve. Each rate leads to a slightly different value for the temperature at which the slope changes. A parameter often used to define the glass transition temperature is the Wendt-Abraham parameter ${ }^{24}$ defined by $R=g_{\min } / g_{\max }$. Here $g_{\min }\left(g_{\max }\right)$ is the value of $g(r)$ at the first minimum (maximum) in the RDF. The Wendt-Abraham parameter stresses the local character of $g(r)$, permitting a direct comparison between structures and leading to a better estimate of glass transition temperatures. The Wendt-Abraham transition temperature, $T_{g}^{\mathrm{WA}}$, is displayed in Fig. 3(b) for each cooling rate. Thus, we see that, $T_{g}^{\mathrm{WA}} \approx 500 \mathrm{~K}$ at $\Delta T / \Delta t=2 \times 10^{12} \mathrm{~K} / \mathrm{s}, \quad T_{g}^{\mathrm{WA}} \approx 550 \mathrm{~K} \quad$ at $\Delta T / d t=4 \times 10^{12} \mathrm{~K} / \mathrm{s}, \quad$ and $T_{g}^{\mathrm{WA}} \approx 700 \mathrm{~K} \quad$ at $\Delta T / \Delta t \approx 4$ $\times 10^{14} \mathrm{~K} / \mathrm{s}$. Thus, the glass transition temperature increases with increased cooling rate. The fastest cooling rates result in shorter times for the atoms to relax, thus leading to formation of the glass at a higher temperature than at lower cooling rate.

\section{B. $\mathrm{CuNi}$}

To contrast with the glass transition observed $\mathrm{CuAg}$ system, we also considered the quenching of liquid $\mathrm{CuNi}$ alloy and pure $\mathrm{Cu}$ using the same cooling rates as for $\mathrm{CuAg}$. 


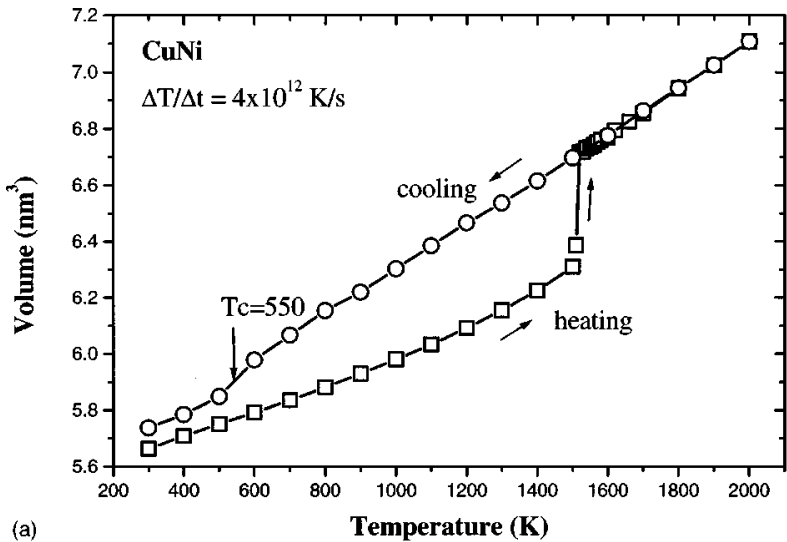

(a)

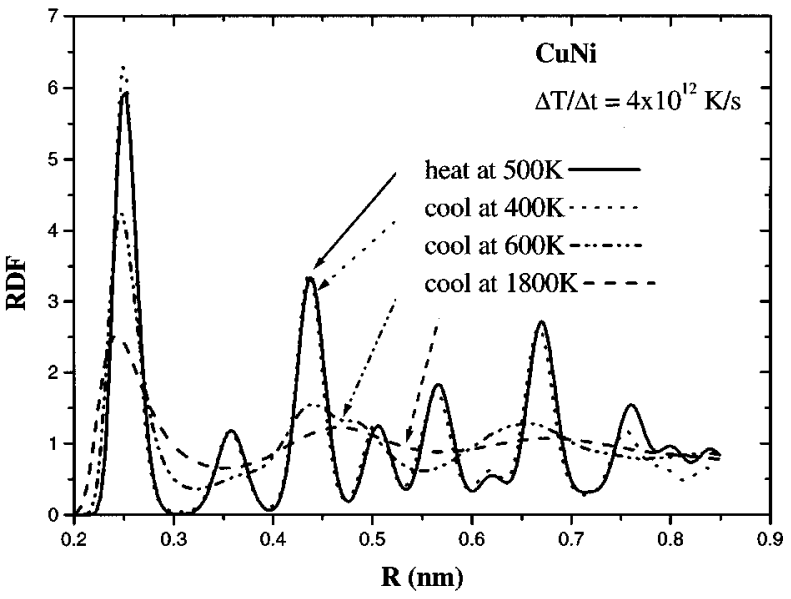

FIG. 5. Melting and cooling (at the quenching rate of 4 $\times 10^{12} \mathrm{~K} / \mathrm{s}$ ) of $\mathrm{NiCu}$ leads to crystallization at $\sim 500 \mathrm{~K}$. (a) Volume versus temperature in heating and cooling cycles. (b) RDF of $\mathrm{NiCu}$ at $500 \mathrm{~K}$ in the heating cycle and at 1800,600 , and $400 \mathrm{~K}$ in the cooling cycle.

The results for $\mathrm{Cu}$ [Fig. 4(a)] show a sharp change in the volume as the temperature is lowered below $600 \mathrm{~K}$. The RDF [Fig. 4(b)] for this system clearly shows that at $500 \mathrm{~K}$ the cooled $\mathrm{Cu}$ has crystallized.

Similarly, cooling the CuNi alloy from the melt [Fig. 5(a)] shows a sharp drop in volume at $500 \mathrm{~K}$. To provide a continuous view of the crystallization in $\mathrm{Cu}$, Fig. 5(b) shows the RDF's calculated at 1800,600 , and $400 \mathrm{~K}$ during the cooling process [and compared with the structure we started (in heating cycle) with at $500 \mathrm{~K}$ ]. The transformation from liquid $(1800 \mathrm{~K})$ to crystal $(400 \mathrm{~K})$ is very clear in Fig. 5 with the peaks formed in cooling well overlapping those from the original crystal in heating cycle.

To understand the progression of melting and crystallization events, we calculated the $S(\mathbf{K})$ from trajectory as a function of time. Figure 6(a) shows the time series for the structure factor calculated for $\mathbf{K}$ vectors $(10,0,0)$ and $(5,5,5)$ from MD runs at 1500,1510 , and $1520 \mathrm{~K}$. The structure factor drops to zero at the same point where the volume and potential energy increase due to melting. Crystallization is the opposite process. The structure factor $S(\mathbf{K})$ will increase as the atoms order in appropriate planes. Since the new crystal need not form in the same orientation of the original box,

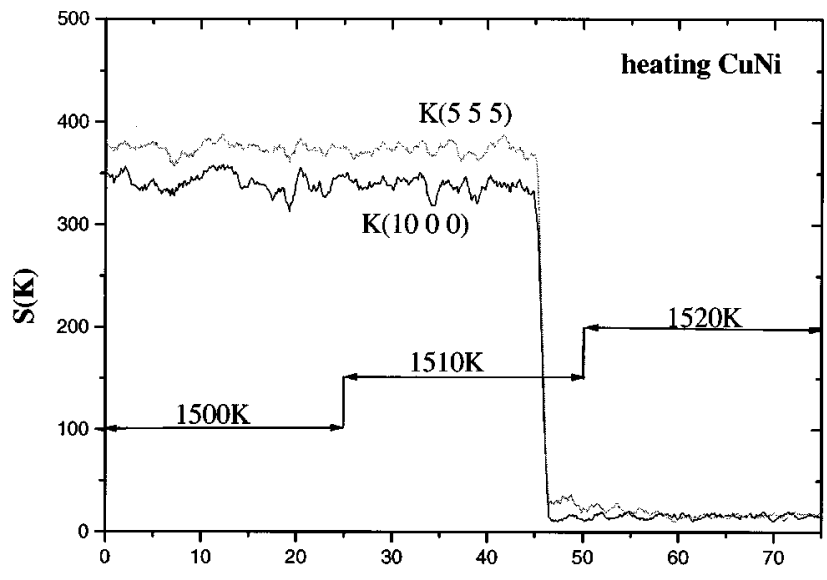

(a)

Time (ps)

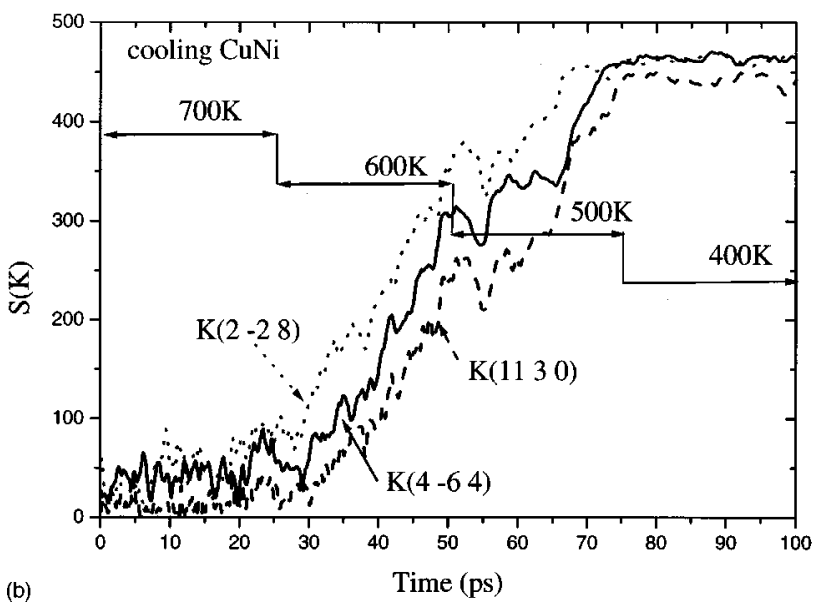

FIG. 6. Structure factor $S(\mathbf{K})$ changes upon melting $\left(T_{m}\right.$ $\sim 1510 \mathrm{~K})$ and crystallization $\left(T_{c} \sim 500 \mathrm{~K}\right)$ in $\mathrm{NiCu}$. (a) The values of $S(\mathbf{K})$ as a function of time for the heating simulations are for 1500 to 1510 to $1520 \mathrm{~K}$. Melting occurs after 21 ps at $1510 \mathrm{~K}$, (b) The values of $S(\mathbf{K})$ as a function of time for the cooling simulations are from 700 to 600 to 500 to $400 \mathrm{~K}$. Crystallization starts at 25 ps and is complete at $75 \mathrm{ps}$.

and the $\mathbf{K}$ vectors here are defined respect to the axes of the box, the $\mathbf{K}$ vectors, on which the $S(\mathbf{K})$ grow, are different with the original ones. Figure 6(b) displays the $S(\mathbf{K})$ values for some $\mathbf{K}$ vectors traced at $700,600,500$, and $400 \mathrm{~K}$. The

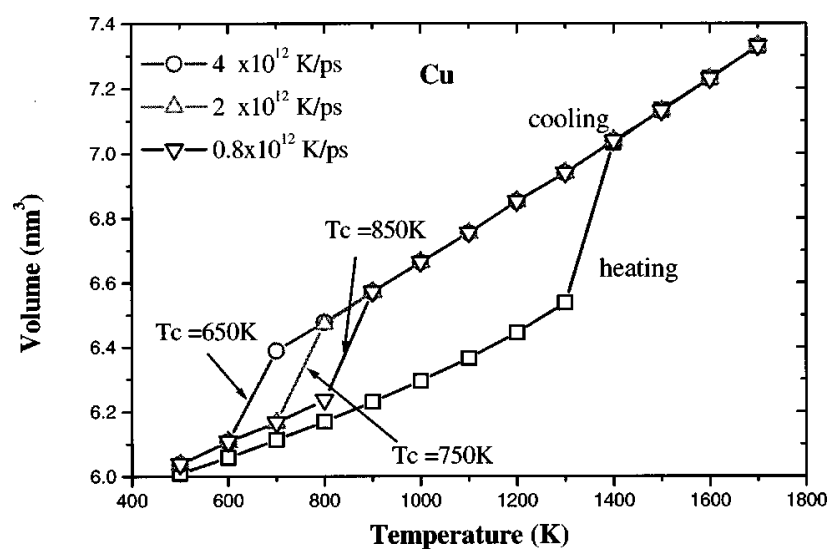

FIG. 7. Effect of cooling rate on crystallization temperature for pure $\mathrm{Cu}$. 

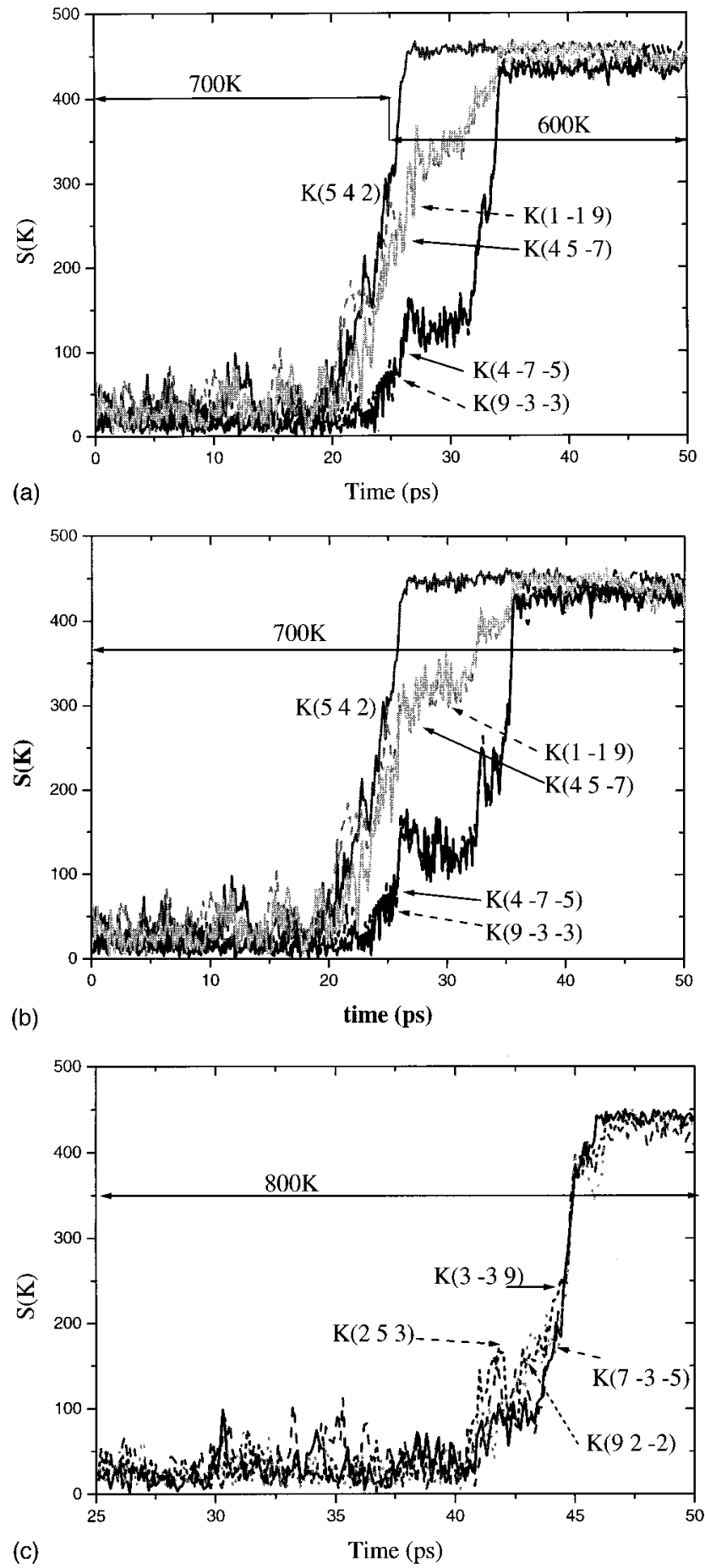

FIG. 8. Change in structure factor for different simulations of crystallization. (a) Normal cooling with 25 ps at $700 \mathrm{~K}$ followed by 25 ps at $600 \mathrm{~K}$. Crystallization is complete at 33 ps. (b) The same 25 ps run at $700 \mathrm{~K}$ followed by second 25 ps again at $700 \mathrm{~K}$. Compared to (a) this shows that the same time is required when the second $25 \mathrm{ps}$ is at 600 or $700 \mathrm{~K}$. Crystallization is complete at 35 ps. The same $\mathbf{K}$ values appear as for case (a) indicating the same nucleus. (c) After the first $25 \mathrm{ps}$ at $800 \mathrm{~K}$, an additional $50 \mathrm{ps}$ were carried out at $800 \mathrm{~K}$ rather than cooling to $700 \mathrm{~K}$ for $25 \mathrm{ps}$ and then cooling to $600 \mathrm{~K}$ for $25 \mathrm{ps}$. The time is referenced to the point at which the normal cycle was dropped to $700 \mathrm{~K}$. Thus, it has the same significance as in (a) and (b). We see that crystallization complete at $T \sim 45 \mathrm{ps}$. The $\mathbf{K}$ values are different than for (a) and (b) indicating a different nucleus. atoms became ordered on specific planes, leading to increase in structure factor for the $\mathbf{K}$ vectors representing these planes. As the nuclei form and grow, these $S(\mathbf{K})$ increase.

We also studied the dependence of crystallization temperature on the cooling rate. Figure 7 shows the volume versus temperature curve for various cooling rates. Here the crystallization temperature $T_{c}$ is the temperature at which the density increases suddenly.

To examine crystallization in detail, we again calculated the structure factor for a set of $\mathbf{K}$ vectors. Using a time increment of $25 \mathrm{ps}$ for each temperature step, the crystallization occurred after $8 \mathrm{ps}$ at $600 \mathrm{~K}$ [based on the drastic increase on $S(\mathbf{K})$ for specific $\mathbf{K}$ vectors] [Fig. 8(a)]. In fact, we see that the nucleus for crystallization had already formed at the end of the $700 \mathrm{~K}$ run. To show this, we followed the 25 ps at $700 \mathrm{~K}$ with another $25 \mathrm{ps}$ simulation at $700 \mathrm{~K}$. We find [Fig. 8(b)] that the structure factor grows to 450 after 10 additional ps (total of $35 \mathrm{ps),} \mathrm{essentially} \mathrm{the} \mathrm{same} \mathrm{as} \mathrm{for} \mathrm{the}$ $650 \mathrm{~K}$ case. The $\mathbf{K}$ vectors in these two runs are identical, indicating that the nucleus had already formed by the end of the first 25 ps at $700 \mathrm{~K}$.

We also carried out a third calculation shown in Fig. 8(c). Here we started with the final structure at $800 \mathrm{~K}$ after 25 ps and continued an additional 50 ps at $800 \mathrm{~K}$. In Fig. 8(c) we denote the start of this last 50 ps as 0 in order to compare with Figs. 8(a) and 8(b). We see that this took 48 ps to crystallize (longer than the 33 or 35 ps for 650 or $700 \mathrm{~K}$ ) but the final crystal has different $\mathbf{K}$ vectors. This indicates that a different nucleus formed.

\section{CONCLUSION}

We applied the Q-SC FF to study the phase transformations in $\mathrm{Ag}_{6} \mathrm{Cu}_{4}$ and $\mathrm{CuNi}$ alloys. We find that $\mathrm{Ag}_{6} \mathrm{Cu}_{4}$ forms a metallic glass for all quenching rates while $\mathrm{Cu}$ and $\mathrm{CuNi}$ form an fcc crystal for all quenching rates. This shows that changing the radius ratio from 1.025 to 1.13 has a dramatic effect on the propensity for glass formation. The observed glass transition temperature and crystallization temperature depend on the quenching rate.

\section{ACKNOWLEDGMENTS}

This research was supported partially by a grant (ARODAAH 95-1-0233) from the Army Research Office and by grants from the NSF (ASC 92-17368 and CHE 95-12279). In addition, support for the Materials Simulation Center (MSC) facilities came from DOE-ASCI, ARO-DURIP (DAAG5597-1-0140), Chevron Petroleum Technology Co, Asahi Chemical, Owens-Corning, Exxon, Asahi Glass, Nippon Steel, Avery Dennison, BP Chemical, and the Beckman Institute. Some calculations were carried out at the Illinois $\mathrm{Na}$ tional Center for Supercomputing Applications (NCSA), funded by the National Science Foundation (NSF). 
*Authors to whom correspondence should be addressed.

${ }^{1}$ P. Duwez, R. H. Willens, and W. Klement, Jr., J. Appl. Phys. 31, 1137 (1960).

${ }^{2}$ A. Peker and W. L. Johnson, Appl. Phys. Lett. 63, 2342 (1993); X. H. Liu and W. L. Johnson J. Appl. Phys. 78, 6514 (1995).

${ }^{3}$ C. Kittel, Introduction to Solid State Physics, 7th ed. (Wiley, New York, 1996) p. 78; W. B. Pearson, Crystal Chemistry and Physics of Metals and Alloys (Wiley, New York, 1972).

${ }^{4}$ H. Choiyim and W. L. Johnson, Appl. Phys. Lett. 71, 3808 (1997); X. Sun, S. Schreider, U. Geyer, W. L. Johnson, and M. A. Nicolet, J. Mater. Res. 11, 2738 (1996).

${ }^{5}$ K. Vollmayr, W. Kob, and K. Binder, J. Chem. Phys. 105, 4714 (1996).

${ }^{6}$ W. C. Swope and H. C. Andersen, Phys. Rev. B 41, 7042 (1990).

${ }^{7}$ M. S. Watanabe and K. Tsumuraya, J. Chem. Phys. 87, 4891 (1987).

${ }^{8}$ H. C. Chen and S. K. Lai, Mater. Sci. Eng., A 179, 261 (1994).

${ }^{9}$ A. C. Brown and R. D. Mountain, J. Chem. Phys. 80, 1263 (1984).

${ }^{10}$ J. Lu and J. A. Szpunar, Philos. Mag. A 75, 1057 (1997).

${ }^{11}$ L. J. Lewis, Phys. Rev. B 39, 12954 (1989).

${ }^{12}$ T. Aihara, K. Aoki, and T. Masumoto, Mater. Sci. Eng., A 179,
256 (1994); T. Aihara, K. Aoki, and T. Masumoto, Mater. Trans., JIM 36, 399 (1995); T. Aihara, Y. Kawazoe, and T. Masumoto, J. Non-Cryst. Solids 207, 875 (1996).

${ }^{13}$ H. Teichler, Phys. Rev. Lett. 76, 62 (1995); H. Teichler, Phys. Rev. E 53, 4287 (1996).

${ }^{14}$ M. Shimono and H. Onodera, Mater. Trans., JIM 39, 147 (1998).

${ }^{15}$ R. G. Dellavalle, D. Gazzillo, and G. Pastore, Mater. Sci. Eng., A 165, 183 (1993).

${ }^{16}$ A. P. Sutton and J. Chen, Philos. Mag. Lett. 61, 139 (1990).

${ }^{17}$ H. Rafii-Tabar and A. P. Sutton, Philos. Mag. Lett. 63, 217 (1991).

${ }^{18}$ Y. Kimura, T. Çă̆ın, Y. Qi, and W. A. Goddard III (unpublished).

${ }^{19}$ Y. Qi, T. Çă̆ın, Y. Kimura, and W. A. Goddard III (unpublished).

${ }^{20}$ J. R. Ray and A. Rahman, J. Chem. Phys. 82, 4243 (1985).

${ }^{21}$ S. Nosè, Mol. Phys. 52, 255 (1984); S. Nosè, J. Chem. Phys. 81, 511 (1984).

${ }^{22}$ M. Parrinello and A. Rahman, Phys. Rev. Lett. 45, 1196 (1980).

${ }^{23}$ M. Parrinello and A. Rahman, J. Appl. Phys. 52, 7182 (1981).

${ }^{24}$ H. R. Wendt and F. F. Abraham, Phys. Rev. Lett. 41, 1244 (1978). 